\title{
QUALIDADE DE VIDA EM EPILEPSIA E PERCEPÇÃO DE CONTROLE DE CRISES
}

\author{
Priscila Camile Barioni Salgado', Elisabete Abib Pedroso de Souza
}

\begin{abstract}
RESUMO - As avaliações afetivo-cognitivas do sujeito são importantes fatores que controlam a maneira como o indivíduo sente o impacto da doença no cotidiano de sua vida. Neste sentido, a percepção de controle de crises é mais importante na avaliação da Qualidade de Vida $(\mathrm{QV})$ do que as variáveis que caracterizam a doença, como sua severidade, tipo, duração e frequência de crises. Este estudo considerou a relação das variáveis subjetivas (percepção de controle de crises) e das características da doença, para avaliar a QV. Foram avaliados 60 sujeitos com diagnóstico de epilepsia, aleatoriamente selecionados no ambulatório de epilepsia do HC/UNICAMP, com idade entre 18 e 70 anos $(M=37,05 ; D P=11,25)$, através do Questionário de Qualidade de Vida 65. As variáveis da doença não apresentaram relação significativa com a QV, com exceção da frequência de crises que apareceu associada à piora na QV, entre crises controladas e crises com frequência acima de 10 por mês $(p=0,021)$. A percepção do controle de crises apareceu significantemente associada à $Q V(p=0,005)$.
\end{abstract}

PALAVRAS-CHAVE: qualidade de vida, percepção de controle de crises, epilepsia.

Quality of life in epilepsy and perception of seizures control

ABSTRACT - The individual affective-cognitive evaluations are important factors that control the way he feels the disease impact in his life. Then, the perception of seizure control is a more important factor to evaluate Quality of Life (QoL) than the illness characteristics, such as the severity, type, sickening period and seizure frequency. This study searched for the relationship among the subjective variables (perception of seizure control) and the illness characteristics to evaluate QoL. The sample consisted of 60 individuals with chronic epilepsy, aging 18 to $70(M=37.05 ; S D=11.25)$, chosen at randon from the ambulatory of epilepsy - HC/ UNICAMP, by the Questionnaire 65. The illness characteristics were not significant, except the seizures frequency, when associated to the impairment in QoL among controlled seizures and seizures with frequency higher than 10 per month $(p=0.021)$. The perception of control was significantly associated to QoL $(p=0.005)$.

KEY WORDS: quality of life, perception of seizures control, epilepsy.

A epilepsia não pode ser considerada um estado, ela é um distúrbio episódico; no entanto, é um distúrbio estigmatizado'. Devinsky e Penry ${ }^{2}$ apontam para a diferenciação existente entre crise e epilepsia. Segundo os autores, a crise tem um limite de duração, ela termina, enquanto que a epilepsia, para muitas pessoas, é uma condição crônica, que limita os pacientes em suas atividades diárias. A epilepsia afeta cada pessoa de forma diferente. Apenas os portadores de epilepsia percebem as limitações e restrições de cada dia. Todas as crises tem um significado dependente da história de vida de cada sujeito, determinando assim padrões de resposta em relação à doença. A epilepsia tem um impacto maior na saúde mental e social do que na saúde física, comparando-a com outras doenças crônicas ${ }^{3}$. Uma do- ença crônica afeta a vida do indivíduo em fatores emocionais, físicos e sociais. Todos estes fatores contribuem para o ajustamento do paciente ao seu distúrbio e sua percepção do impacto na sua qualidade de vida $(Q V)^{4}$. Segundo Anderson e Bury ${ }^{5}$, um distúrbio crônico é um processo de ajustamento e mudança contínuo.

Entende-se como QV a perspectiva do sujeito que avalia como vê e sente a interferência da doença na sua vida pessoal, familiar e profissional ${ }^{6}$. O paciente portador de epilepsia tem a percepção de sua doença através de suas crises. Ele pode não ter mais crises, mas se ainda se preocupa em tê-las, sua QV é afetada da mesma forma. O que importa é como o indivíduo se percebe. Assim, as avaliações afetivocognitivas do sujeito são importantes fatores que

Faculdade de Ciências Médicas (FCM) da Universidade Estadual de Campinas (UNICAMP) Campinas SP, Brasil: 'Psicóloga, Mestranda em Ciências Médicas FCM/UNICAMP, Área de Concentração Neurologia; 2 Psicóloga, Professor Assistente Doutor, Departamento de Neurologia da FCM/UNICAMP.

Recebido 22 Novembro 2000, recebido na forma final 26 Março 2001. Aceito 11 Abril 2001.

Dra. Elisabete Abib Pedroso de Souza - Departamento de Neurologia FCM/UNICAMP - Caixa Postal 6111 - $13083-970$ Campinas SP Brasil.E-mail: assouza@bestway.com.br 
controlam a maneira como ele sente o impacto da doença em seu dia-a-dia. Neste sentido, a percepção de controle de crises é mais importante do que as variáveis da doença (tipo de crise, frequência, início, duração) na avaliação da QV7-8.

Este estudo objetivou avaliar a QV em portadores de epilepsia e relacionar com variáveis do sujeito (percepção de controle de crise) e variáveis da doença (tipo de crise, frequência, início, duração).

\section{MÉTODO}

\section{Sujeitos}

Foram avaliados 60 pacientes com epilepsia diagnosticada há mais de três anos, selecionados aleatoriamente no Ambulatório de Epilepsia do HC/Unicamp. A amostra constou de pacientes adultos com idade entre 18 e 70 anos, $(M=37,05 ; D P=11,25)$. A maioria dos sujeitos possuía $1^{\circ}$ grau incompleto (52,6\%), não tinha nenhum vínculo empregatício $(72,9 \%)$, era solteira $(50,8 \%)$ e não tinha filhos $(53,3 \%)$

Quanto à epilepsia, a idade de início das crises variou de 0 a 48 anos $(M=11,17 ; D P=10,86)$ e a duração da doença variou de 4 a 48 anos $(M=25,68$; $D P=11,28)$. Quanto ao tipo de crise, 43,9\% dos sujeitos apresentavam crises parciais secundariamente generalizadas (CP-G), $35,1 \%$, crises tônico clônico generalizadas (CTCG) e 21,0\% crises parciais (CP). Quanto à freqüência de crises, 23,3\% dos sujeitos apresentavam controle de crises há mais de seis meses, 55,0\% apresentavam de 1 a 10 crises por mês e $21,7 \%$, de 11 a 30 . Quanto à percepção de controle de crises, $50,0 \%$ dos sujeitos consideraram que suas crises estavam controladas.

Foram excluídos pacientes com retardo mental evidente ou doenças psiquiátricas e sob medicação que não drogas anti-epilépticas que pudessem afetar o sistema nervoso central.

\section{Intrumentos}

Foi utilizada uma ficha de identificação com dados demográficos (nome, idade, escolaridade, emprego, estado civil) e dados da doença (início, duração, tipo, freqüência, severidade, medicação).

O Questionário de Qualidade de Vida 65 (QQV-65), foi aplicado em cada paciente, através de uma entrevista clínica. 0 questionário contém 65 itens que avaliam a percepção de saúde e QV (10 itens), limitação física (5 itens), aspectos sociais (6 itens), satisfação (6 itens), percepção de controle (6 itens), aspectos afetivo-emocionais (12 itens), auto-conceito (8 itens), aspectos cognitivos (4 itens), trabalho (5 itens), lazer (2 itens) e dirigir veículos ( 1 item). A avaliação de QV foi através da pontuação total no teste QQV-65.

O QQV-659 foi elaborado levando em consideração as restrições linguísticas para a população específica, relativas às dificuldades de compreensão das questões e confusão na discriminação de diferentes intensidades de res- postas relacionadas sensivelmente a déficits de escolaridade. O QQV-65 é um instrumento utilizado na rotina de atendimento aos pacientes portadores de epilepsia no setor de Psicologia aplicada à Neurologia, do HC-UNICAMP.

A informação de percepção de controle de crises foi obtida através do relato dos pacientes que avaliaram ter ou não suas crises controladas. Este é um dado subjetivo, que se refere à avaliação do sujeito a respeito de como ele percebe o controle das crises. É diferente da frequência de crises, que se refere ao número real de crises expresso em dias, meses ou anos.

\section{Procedimento}

Após a seleção e avaliação médica neurológica, que consta de uma anamnese detalhada, exame físico geral, exame neurológico e exames complementares, como eletroencefalograma (EEG), tomografia computadorizada de crânio (TCC) e ressonância magnética (RM), os sujeitos foram encaminhados ao setor de Psicologia.

A avaliação psicológica constou de entrevista não estruturada e aplicação do teste da pesquisa. Após a aplicação do QQV-65, os pacientes foram encorajados a falar sobre qualquer outro aspecto que julgassem importante.

\section{Análise estatística dos dados}

Para caracterizar a casuística foram utilizadas tabelas de frequência (frequência relativa e frequência absoluta) para descrever as variáveis categóricas (sexo, escolaridade, tipo de emprego, estado civil, religião, tipo de crises) e estatísticas descritivas ( média, desvio padrão, mediana e valores máximo e mínimo) para as variáveis contínuas (pontuação de QV, frequência de crises, idade, idade de início das crises, duração de crises, fator escola, fator trabalho, fator saúde física, fator relacionamento social, fator lazer, fator saúde emocional, percepção de controle, auto-conceito).

Cada uma das pontuações foi comparada com outras variáveis do questionário. Se a variável foi medida em escala contínua (valores numéricos), foi calculado o coeficiente de correlação de Spearman. Se a variável foi medida em escala categórica, foi utilizado o teste não-paramétrico de Mann-Whitney (quando comparando apenas duas categorias) ou o teste de Kruskall-Wallis ( para três ou mais categorias).

Para cruzamento entre duas variáveis categóricas, foi utilizado o teste de associação QUI-quadrado ou quando necessário, o teste exato de Fisher.

\section{RESULTADOS}

A análise da relação entre idade de início e duração das crises com QV mostrou independência entre as variáveis $(p=0,664$ e $p=0,892$; coeficientes da correlação de Spearman).

Não houve associação entre QV e tipo de crise $(p=0,288$; Kruskal-Wallis), mostrando independência entre as variáveis (Fig 1). 


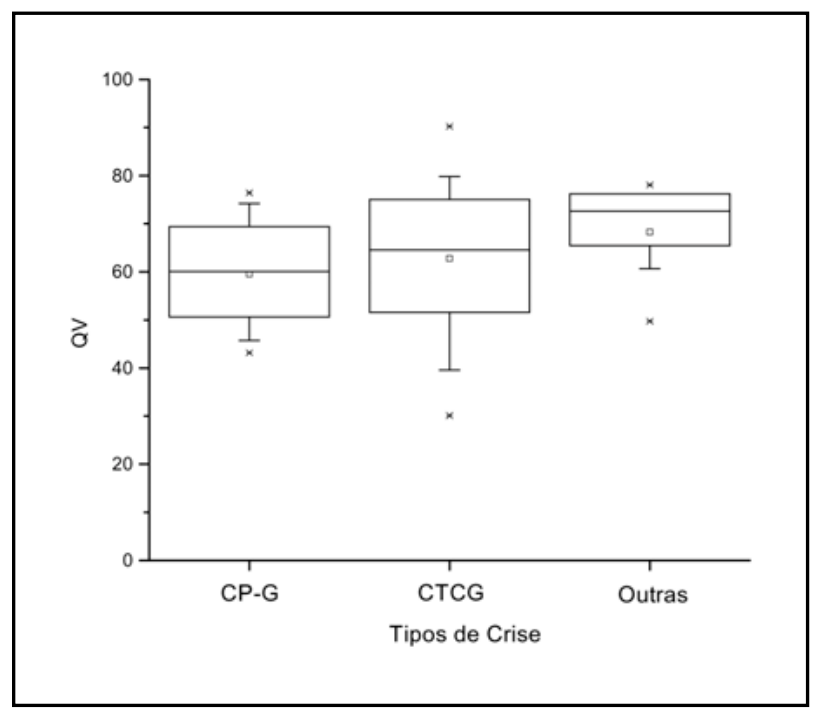

Fig 1. Boxplot da qualidade de vida em função do tipo de crise.

Tabela 1. Resultados de significância relativa às variáveis da doença e variável do sujeito.

\begin{tabular}{lc}
\hline & $p$-valor \\
\hline Idade de início das crises & 0,664 \\
Duração das crises & 0,892 \\
Tipo de crise & 0,288 \\
Freqüência de crise & 0,021 \\
Percepção de controle de crise & 0,005 \\
\hline
\end{tabular}

A análise da relação entre $Q V$ e frequência de crise foi significativa. A frequência apareceu associada à piora na QV quando se compararam crises controladas (mais de 150 dias sem crise) e crises com freqüência acima de 10 por mês ( $p=0,021$; KruskalWallis) (Fig 2).

$\mathrm{O}$ teste de Mann-Whitney mostrou haver relação entre a percepção de controle de crises e QV. Foi observada associação altamente significativa $(p=0,005)$ (Fig 3).

A Tabela 1 mostra os valores de $p$ para cada aspecto analisado.

\section{DISCUSSÃO}

Os dados mostram a importância das variáveis subjetivas controlando as respostas de bem-estar. A maneira como julgamos uma situação está relacionada à interpretação e às expectativas que temos dela, o que depende da história de vida do indivíduo e de estratégias que este utiliza para ajustar-se à sua condição de saúde. O indivíduo cria teorias,

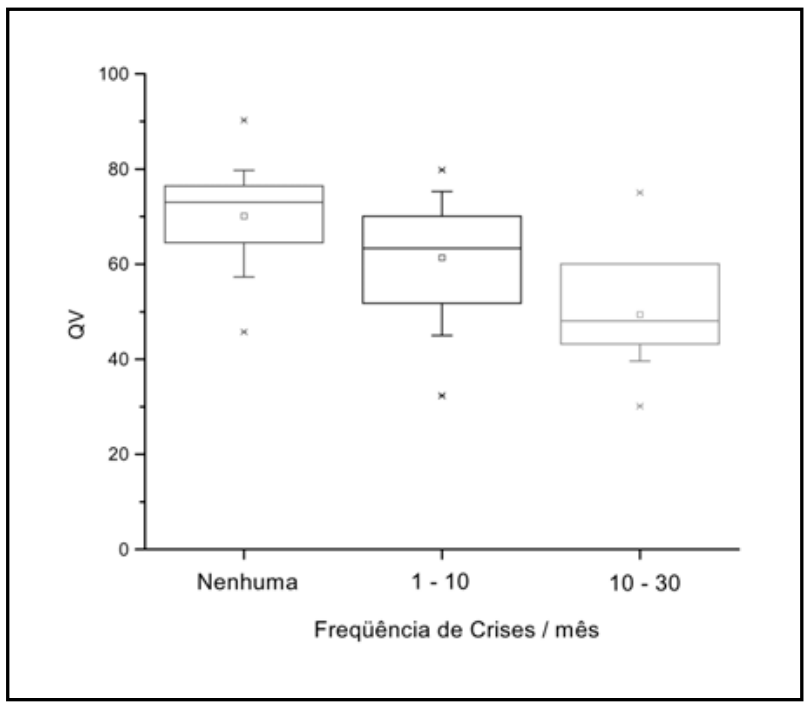

Fig 2. Boxplot da qualidade de vida em função da frequência de crises.

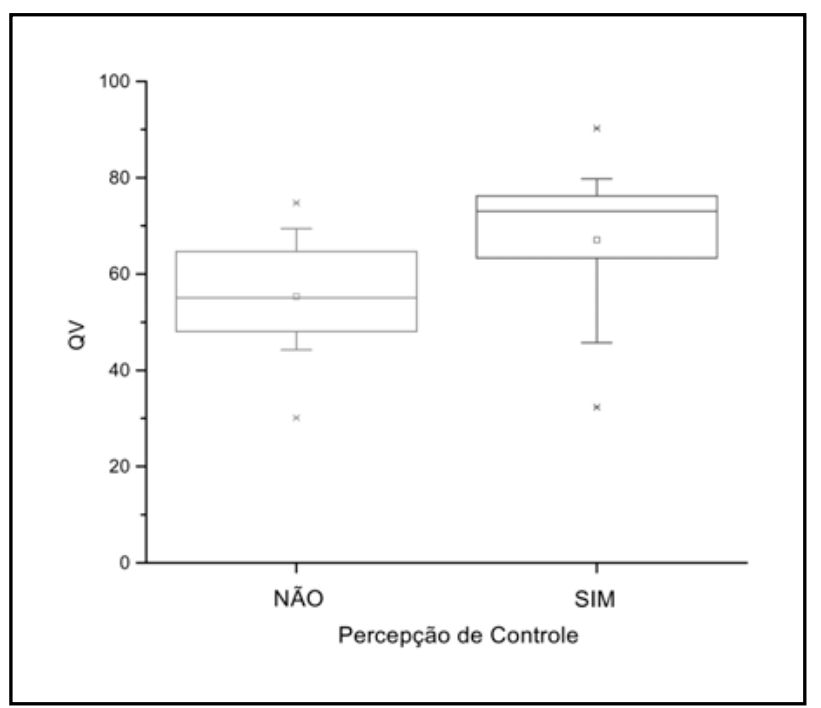

Fig 3. Boxplot da qualidade de vida em função da percepção de controle.

explicações e diferentes valores para a situação em questão. Assim, o significado afetivo e cognitivo da informação, que inicialmente pode ter sido experienciada como ameaçadora, pode mudar, tornando a situação mais aceitável ${ }^{10}$. É o significado afetivocognitivo de determinada situação que controla o nível de sentimentos e comportamentos subsequentes.

O desenvolvimento de uma doença crônica modifica a maneira como o indivíduo se percebe, modifica sua vida social e econômica e seus planos de futuro. Em geral, a percepção de bem-estar de uma pessoa portadora de uma doença crônica sofre mais influência dos mecanismos da auto-avaliação do que significa estar doente, do que do próprio estado fí- 
sico. Estes mecanismos de avaliação e estratégias de adaptação psicológica são fundamentais na manutenção de níveis aceitáveis de bem-estar diante do distúrbio crônico. A percepção do controle de crises sofre influência destes mecanismos de avaliação e explica os resultados encontrados neste estudo, referentes à associação altamente significativa encontrada entre QV e percepção do sujeito quanto ao controle de suas crises.

A QV sofre influência de vários fatores relacionados a: estratégias de adaptação psicossocial ${ }^{10}$, traços de personalidade do indivíduo ${ }^{11}$, sua história e expectativas em relação a vida ${ }^{12}$, seu auto-conceito ${ }^{10}$ e auto-eficiência ${ }^{13}$, suporte social que recebe das pessoas que the são significativas ${ }^{13}$, seu locus de controle ${ }^{10-13-14-15}$ e percepção de controle de crises. Dentre estes fatores, esta última foi a variável subjetiva pesquisada neste estudo.

O locus de controle pode ser definido como a percepção que o paciente tem de causalidade. 0 locus de controle interno indica a percepção da habilidade em controlar os eventos da vida, enquanto que o externo indica que a pessoa acredita que sua vida é controlada por forças como destino e sorte. Geralmente, pacientes portadores de epilepsia têm um locus de controle externo, pois acreditam que têm pouco controle de sua doença e de sua vida. A experiência de falta de controle e incerteza é central para estes pacientes. Isto se aplica às crises, falta de controle do próprio corpo, dependência da medicação e de ajuda dos outros significativos ${ }^{13}$. Pessoas com epilepsia generalizam seus sentimentos de falta de controle durante a crise para outros aspectos de suas vidas e desenvolvem uma expectativa de que certas experiências estão além de seu controle. Há associação entre a falta de controle nas crises e outros aspectos da vida. O locus de controle externo está associado à psicopatologia, principalmente à depressão ${ }^{14}$ e à dificuldade de ajustamento psicossocial.

A investigação de locus de controle focalizou neste trabalho a percepção do indivíduo em relação ao controle de crises. A maneira como o sujeito percebe suas crises é uma medida subjetiva que con- trola sua resposta de bem-estar. A percepção de ter as crises controladas ou não pareceu ser altamente relacionada à QV do sujeito e foi mais importante do que as variáveis da doença (tipo de crise, freqüência, início, duração) na avaliação da QV.

A redução na freqüência de crises epilépticas não produziu necessariamente uma melhora na QV do paciente, o que reforça o conceito de que medidas subjetivas controlam satisfação e bem-estar.

Agradecimentos - Agradecemos a colaboração dos Profs. Drs. Carlos A.M. Guerreiro e Fernando Cendes do Departamento de Neurologia da FCM/UNICAMP responsáveis pelo ambulatório de epilepsia, bem como de Helymar da Costa Machado, Mestre, pelo tratamento estatístico. Comissão de Estatística da FCM/UNICAMP.

\section{REFERÊNCIAS}

1. Cramer JA. A Clinimetric Approach to Assessing Quality of Life in epilepsy. Epilepsia 1993;34 (Suppl. 4):S8-S13.

2. Devinsky O, Penry JK. Quality of Life in Epilepsy: The Clinician's View. Epilepsia 1993;34 (Suppl. 4):S4-S7.

3. Vickrey BG. A procedure for developing a Quality-of-Life measure for epilepsy surgery patients. Epilepsia 1993;34 (Suppl. 4):S22-S27.

4. Jacoby A, Baker G, Smith D, Dewey M, Chadwick. Measuring the impact of epilepsy: the development of a novel scale. Epilepsy Res 1993; 16:83-88.

5. Anderson R, Bury M. Living with chronic illness: the experience of patients and their families. London: Allen \& Unwin, 1988.

6. Souza EAP, Guerreiro MM. Qualidade de Vida. In Guerreiro CAM, Guerreiro MM (eds). Epilepsia. São Paulo: Lemos, 1996;191-199.

7. Baker GA, Gagnon D, McNulty P. The relationship between seizure frequency, seizure type and QoL: findings from three European countries. Epilepsy Res 1998;30:231-240.

8. Smith DF, Baker GA, Dewey M, Jacoby A, Chadwick DW. Seizure frequency, patient-perceived seizure severity and the psychosocial consequences of intractable epilepsy. Epilepsy Res 1991;9:231-241.

9. Souza EAP, Veiga BA, Guerreiro MM, Cendes F. Desenvolvimento de protocolo de Qualidade de Vida (QV) para pacientes epilépticos. Braz J Epilepsy Clin Neurophysiol 1998;4:92.

10. Gertrudis IJM, Kempen PRD, Ormel J, Brilman I, Relyveld J. Adaptative responses among Dutch elderly: the impact of eight chronic medical conditions on HRQOL. Am J Public Health 1997;87:38-44.

11. Rose KJ, Derry PA, McLachlan RS. Neuroticism in Temporal lobe Epilepsy: Assessment and implications for pre-and postoperative psychosocial adjustment and health-related quality of life. Epilepsia 1996; 37:484-491.

12. Calman KC. Quality of Life in cancer patients an hypothesis. J Med Ethics 1984;10:124-127.

13. Amir M, Roziner I, Knoll A, Neufeld MY. Self-efficacy and social support as mediators in the relation between disease severity and quality of life in patients with epilepsy. Epilepsia 1999;40:216-224.

14. Hermann BP, Whitman S, Wyller AR, Anton MT, Vanderzwagg R. Psychosocial Predictors of Psychopatology in Epilepsy. Brit J Psych 1990;156:98-105

15. Gopinath B, Radhakrishnan K, Sarma PS, Jayachandran D, Alexander A. A questionnaire survey about doctor-patient communication, compliance and locus of control among South Indian people with epilepsy. Epilepsy Res 2000;39:73-82. 